Check for updates

Cite this: RSC Adv., 2019, 9, 29942

Received 8th August 2019

Accepted 16th September 2019

DOI: 10.1039/c9ra06164a

rsc.li/rsc-advances

\section{A highly sensitive fluorimetric protocol based on isoindole formation for determination of gabapentin}

\begin{abstract}
Tamer Z. Attia, (D) Mohamed Elnady and Sayed M. Derayea (D)*
A new, simple, highly sensitive and selective spectrofluorimetric method was developed for determination of gabapentin through its derivatization with o-phthalaldehyde in the presence of 2-mercaptoethanol. The resulting product was highly fluorescent and its emission intensity was measured at $431 \mathrm{~nm}$ after excitation at $335 \mathrm{~nm}$. The effect of different experimental parameters on the formation and stability of the fluorescent product was carefully studied and optimized. The fluorescence-concentration plot was rectilinear over the range of 25-125 $\mathrm{ng} \mathrm{mL}$. The lower detection and quantification limits were $3.4 \mathrm{~mL}^{-1}$ and $11.2 \mathrm{ng} \mathrm{mL}^{-1}$, respectively. The procedure was fully validated according to the guidelines of the International Conference on Harmonization. The proposed method was successfully applied for the determination of the investigated drug in its pharmaceutical capsules and the results were in agreement with those of the reported method, in terms of the accuracy and precision. The low cost of analysis and high sensitivity make the proposed method ideally suited for analysis of the investigated drug in quality control laboratories.
\end{abstract}

\section{Introduction}

Gabapentin (Fig. 1), chemically known as 1-(amino methyl) cyclohexane acetic acid, is a structural analogue of the inhibitory neurotransmitter gamma-amino butyric acid (GABA) and its action is attributed to the irreversible inhibition of the enzyme GABA-transaminase, thus preventing the physiological degradation of GABA in the brain. It is an anticonvulsant drug used in the treatment of epilepsy and neuropathic pain, as an adjunct therapy for partial seizures in adults and children. ${ }^{1}$

An analytical literature survey revealed that several methods have been reported for determination of gabapentin in pure forms and pharmaceutical dosage forms including spectrophotometry, ${ }^{2-4}$ spectrofluorimetry, ${ }^{5-8}$ high-performance liquid chromatography (HPLC), ${ }^{9-11}$ gas chromatography-mass spectrometry (GC-MS), ${ }^{12}$ capillary electrophoresis ${ }^{13}$ and electrochemical techniques. ${ }^{14}$

The reported spectrofluorimetric methods of analysis are either suffer from low sensitivity ${ }^{5-7}$ or require heating and tedious extraction procedures. ${ }^{8}$ In addition, the chromatographic, electrophoretic and electrochemical methods require highly sophisticated instruments and/or require expensive detector which is not available in most laboratories.

The aim of the present work is to develop a simple and highly sensitive spectrofluorimetric method for determination of gabapentin. The amino group of the investigated drug was condensed with $o$-phthalaldehyde to form a highly fluorescent

Department of Analytical Chemistry, Faculty of Pharmacy, Minia University, Minia, Egypt.E-mail: sayed_derayea@mu.edu.eg product. The proposed method was applied for the determination of gabapentin in its capsules without any interference from the excipients. The advantage of spectrofluorimetric method over other methods of analysis is that it does not require highly sophisticated instruments and more sensitive than the reported spectrophotometric methods of analysis.

\section{Experimental}

\subsection{Apparatus}

PerkinElmer UK model LS 45 Luminescence Spectrometer was used for performing all the spectrofluorimetric measurements. The instrument is equipped with a $150 \mathrm{~W}$ xenon arc lamp and a $1 \mathrm{~cm}$ quartz cell, connected to an IBM PC computer loaded with the FL WINLAB ${ }^{\mathrm{TM}}$ software. Grating excitation and emission monochromators slit width for both monochromators were set at $10 \mathrm{~nm}$.

Spectronic ${ }^{\mathrm{TM}}$ Genesys $^{\mathrm{TM}}$ 2PC Ultraviolet/Visible Spectrophotometer (Milton Roy Co, USA) with a matched $1 \mathrm{~cm}$ quartz cell, connected to IBM computer loaded with Winspec ${ }^{\mathrm{TM}}$ application software. Milwaukee SM $101 \mathrm{pH}$ meter, Portugal and Digital Analytical Balance (AG 29, Mettler Toledo, Glattbrugg, Switzerland) were also used.

\subsection{Chemicals and reagents}

Gabapentin was kindly provided by Delta Pharm Company $\left(10^{\text {th }}\right.$ of Ramadan city, El Sharkeya, Egypt). It was used without further purification. $o$-Phthalaldehyde (Loba Chemie, Pvt. Ltd. 107, Woodhouse road, Mumbai, India) was freshly prepared by 
<smiles>O=Cc1ccccc1C=O</smiles>

ofhthalaldehyde

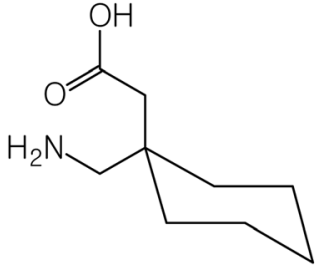

Gabapentin

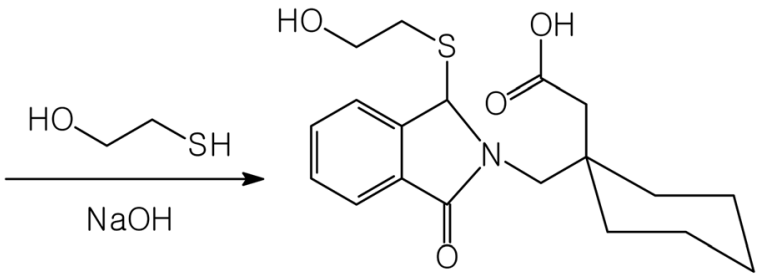

Isoindole derivative

Fig. 1 The proposed pathway of the reaction between gabapentin and o-phthalaldehyde/2-mercaptoethanol reagent.

dissolving $10 \mathrm{mg}$ in $3 \mathrm{~mL}$ of methanol and complete to $100 \mathrm{~mL}$ with distilled water. Working solution of the reagent $(1 \mu \mathrm{g}$ $\mathrm{mL}^{-1}$ ) was prepared by further dilution with distilled water. 2Mercaptoethanol (Alpha Chemika, Mumbai, India) was freshly prepared as $0.5 \%(\mathrm{v} / \mathrm{v})$ in water. Sodium hydroxide (El-Nasr Chemical Co., Cairo, Egypt) $(0.1 \mathrm{M})$ was prepared by dissolving $400 \mathrm{mg}$ in $100 \mathrm{~mL}$ of distilled water. Methanol was obtained from Merck, Darmstadt, Germany. All the reagents used were of analytical grade and the solutions were prepared with double distilled water.

\subsection{Pharmaceutical formulations}

The following available commercial preparation was analyzed; Gaptin ${ }^{\circledR}$ capsules (Delta Pharma S.A.E, $10^{\text {th }}$ of Ramadan City, El Sharkeya, Egypt) labeled to contain $100 \mathrm{mg}$ gabapentin per capsule.

\subsection{Preparation of standard solution}

A stock solution of gabapentin was prepared by dissolving $10.0 \mathrm{mg}$ of the investigate drug in $100 \mathrm{~mL}$ methanol. This solution was further diluted with the same solvent as appropriate to obtain the required working concentrations. The standard solutions were stable for at least 7 days when kept in the refrigerator.

\subsection{General analytical procedure}

Aliquots of gabapentin standard solutions covering the working concentration ranges (25-125 $\mathrm{ng} \mathrm{mL}^{-1}$ as final concentrations) were quantitatively transferred into a series of $10 \mathrm{~mL}$ volumetric flasks. To each flask $0.6 \mathrm{~mL}$ of 2-mercaptoethanol solution $(0.5 \% \mathrm{v} / \mathrm{v})$ was added, followed by $0.8 \mathrm{~mL}$ of $0.1 \mathrm{M}$ sodium hydroxide and mixed well. The solutions were allowed to stand for $10 \mathrm{~min}$. Then, $1.7 \mathrm{~mL}$ of $o$-phthalaldehyde was added. The reaction mixture was allowed to stand for $25 \mathrm{~min}$. The volume was completed to the mark with methanol and the fluorescence intensity of the reaction product was measured at $431 \mathrm{~nm}$ after excitation at $335 \mathrm{~nm}$. The relative fluorescence intensity was plotted against the final concentration of the drug $\left(\mathrm{ng} \mathrm{mL}^{-1}\right)$ to get the calibration graph.

\subsection{Analysis of pharmaceutical formulations}

Twenty capsules containing the investigated drug were accurately weighed and mixed thoroughly. An amount of the powder equivalent to $10 \mathrm{mg}$ of the drug was dissolved in $100 \mathrm{~mL}$ methanol. The solution was further diluted with the same solvent and a portion of the resulting solution was subjected for drug analysis using the previously described general analytical procedure. The nominal content of the capsules were calculated using the corresponding regression equation.

\section{Result and discussion}

$o$-Phthalaldehyde in combination with a thiol compound, such as 2-mercaptoethanol, is widely utilized as a fluorescence derivatizing agent for amino compounds. This approach has been applied for the determination of several pharmaceutical compounds. ${ }^{15-17}$ A condensation reaction occurs between the aldehydic groups of $o$-phthalaldehyde and primary amine group of gabapentin lead to formation of a high fluorescent reaction product. Addition of 2-mercaptoethanol to the solution is essential to stabilize the formed reaction product. A proposal for the reaction pathway is presented in Fig. 1. The formed product exhibited high fluorescence at $431 \mathrm{~nm}$ after excitation at $335 \mathrm{~nm}$. The fluorescence spectra of the reaction product obtained by using $75 \mathrm{ng} \mathrm{mL} \mathrm{m}^{-1}$ of gabapentin against the blank treated in the same manner are shown in Fig. 2.

The developed spectrofluorimetric method has several advantages over the previous reported methods. The current spectrofluorimetric method is characterized by high sensitivity

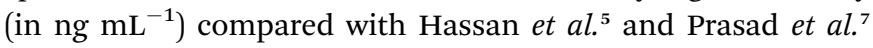
methods. Also, the method is cheap as it does not depend on using expensive reagent compared with Belal et al. method. ${ }^{6}$ In addition, the proposed spectrofluorimetric method is highly simple as there is no need for heating, using buffer or extraction with ethyl acetate as in Ulu et al. method. ${ }^{8}$ As a result, the current spectrofluorimetric method is a good alternative to the previous reported methods and could be ideally suited for quality control laboratory.

\subsection{Optimization of the reaction condition}

The fluorescence properties of the reaction product, as well as the different experimental parameters affecting the development and stability of the reaction product were carefully studied and optimized. Each factor was changed individually while others were kept constant. The examined factors included; volumes of 2-mercaptoethanol, sodium hydroxide and $o$ phthalaldehyde, reaction time and diluting solvents.

3.1.1 Effect of volume of 2-mercaptoethanol. The addition of 2-mercaptoethanol is necessary to stabilize the reaction 


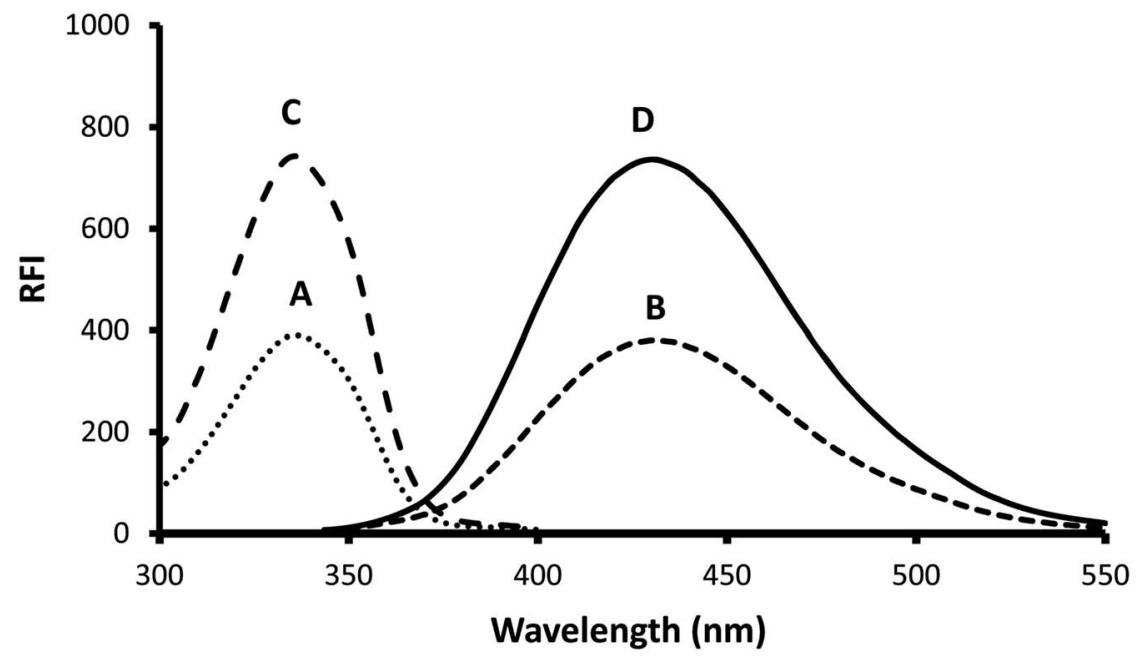

Fig. 2 The fluorescence spectra of the reaction product by using $75 \mathrm{ng} \mathrm{mL}^{-1}$ gabapentin. Where, A (round dot line): excitation of the blank, B (square dot line): emission of the blank, $C$ (dash line): excitation of the reaction product, and $D$ (solid line): emission of the reaction product.

product of the studied drug with $o$-phthalaldehyde. Different volumes of $0.5 \%$ 2-mercaptoethanol solution $(0.2-1.2 \mathrm{~mL})$ were used in performing the general analytical procedure. It was found that, increasing the volume of the reagent produces a proportional increase in the fluorescence intensity of the reaction product up to $0.4 \mathrm{~mL}$. The fluorescence intensity remains unchanged up to $0.8 \mathrm{~mL}$ (Fig. 3). Therefore, $0.6 \mathrm{~mL}$ of $0.5 \%$ of 2-mercaptoethanol solution was chosen as the optimal volume of the reagent.

3.1.2 Effect of sodium hydroxide volume. The fluorescence intensity of the reaction products of the investigated drug was examined using different volumes $(0.2-1.5 \mathrm{~mL})$ of $0.1 \mathrm{M}$ sodium hydroxide solution. Increasing the reagent volume resulted in increasing the fluorescence intensity. Maximum values were obtained at $0.5 \mathrm{~mL}$ of sodium hydroxide and remain constant up to $1.0 \mathrm{~mL}$. However, higher volumes of the reagent gradually decreased the fluorescence intensity of the reaction products. Therefore, $0.8 \mathrm{~mL}$ of sodium hydroxide was selected as optimum volume throughout the study (Fig. 3).

3.1.3 Effect of volume of $o$-phthalaldehyde. The influence of changing $o$-phthalaldehyde volume was studied using different volumes $(0.5-2.5 \mathrm{~mL})$ of the reagent (Fig. 4). It was observed that, increasing the volume of the reagent produces a proportional increase in the fluorescence intensity of the

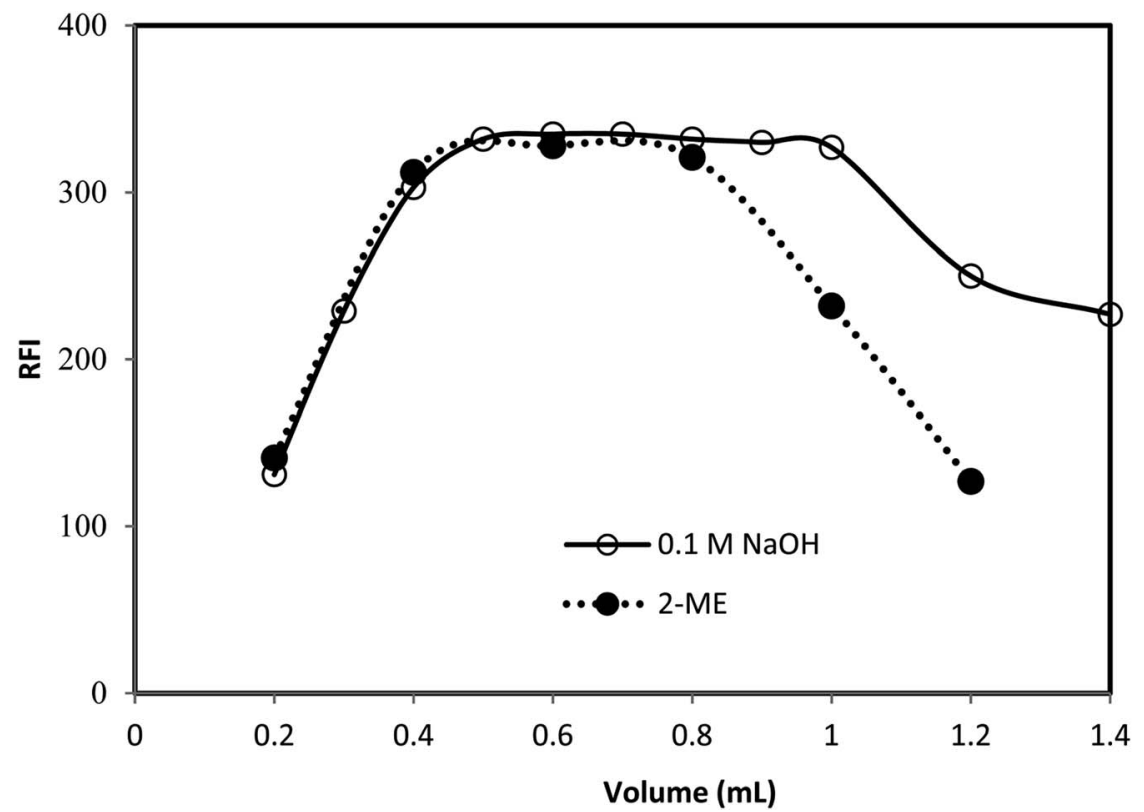

Fig. 3 Effect of the volume of $0.5 \%$ v/v 2 -mercaptoethanol (using $0.8 \mathrm{~mL}$ of $0.1 \mathrm{M} \mathrm{NaOH}$ ) and effect of volume of $0.1 \mathrm{M} \mathrm{NaOH}$ (using $0.6 \mathrm{~mL}$ of $0.5 \% \mathrm{v} / \mathrm{v} 2$-mercaptoethanol) on the relative fluorescence intensity of the reaction product of $75 \mathrm{ng} \mathrm{mL}^{-1} \mathrm{gabapentin}$ 
reaction product up to $1.6 \mathrm{~mL}$. The fluorescence intensities remained unchanged until $1.8 \mathrm{~mL}$ after which, a gradual decrease in the fluorescence intensity were observed. Consequently, $1.7 \mathrm{~mL}$ of $o$-phthalaldehyde solution was chosen as the optimal.

3.1.4 Effect of reaction time. The reaction was allowed to proceed at different time intervals were tested (Fig. 4) and it was found that maximum relative fluorescence intensity was obtained after $20 \mathrm{~min}$ and remains stable up to $30 \mathrm{~min}$. Hence, allowing the reaction mixture to stand for 25 min was adequate for maximum fluorescence intensity.

As, the fluorescence intensity is directly proportional to the fluorescent product concentration. By increasing the reaction time, volume of 2-mercaptoethanol, sodium hydroxide or $o$ phthalaldehyde, the concentration of the fluorescent product (fluorophore) increase and subsequently the fluorescent intensity increased up to saturation. Further increasing in the fluorophore concentration, the fluorophore absorbs the exciting and possibly emitted light (the inner filter effect), reducing the amount of light detected and hence reduces the fluorescence intensity.

3.1.5 Effect of diluting solvent. The formed fluorophore was diluted with different solvents. The examined solvents included; water, methanol, ethanol, isopropyl alcohol, dimethyl formamide and acetone (Fig. 5). Of all the studied solvents, the highest fluorescence intensity was obtained upon using methanol. The variable effect of different types of solvents on the fluorescence intensity of the formed product could be attributed to that the changes in the solvent polarity and the surrounding environment have a great effect on the formed fluorophore.

\subsection{Validation of the proposed method}

According to ICH Q2 recommendations, ${ }^{18}$ the analytical validity of the method was tested regarding; linearity, sensitivity, accuracy, and precision.

Linearity and range: the calibration curve for studied drug was constructed by plotting the relative fluorescence intensities (RFI) against the final investigated drug concentrations (ng $\mathrm{mL}^{-1}$ ). Linear regression analysis of the data was performed and the calculated analytical parameters including slope, intercept, standard deviation of slope and intercept and correlation coefficient are summarized in Table 1. From the calibration curves, we could observe that the RFI values and the drug concentrations was linear dependent within the range of 25-125 ng $\mathrm{mL}^{-1}$ of gabapentin. The excellent linearity of the proposed method was indicated by the high correlation coefficients. In addition, the small values of standard deviation of the intercept $\left(S_{\mathrm{a}}\right)$, and standard deviation of the slope $\left(S_{\mathrm{b}}\right)$ indicate low scattering of the points around the calibration curves.

Accuracy: in general, the accuracy could be defined as the closeness or agreement of the obtained analytical value to the true value and measured by calculating the percentage (\%) recoveries. For the proposed method, the accuracy was checked by preparation of three standard solutions containing different concentrations of the studied drug within the specified linear range. The concentration of each standard solution was measured by our proposed method in triplicate manner. As shown in Table 2, the obtained \% recoveries are close to $100 \%$ which indicates the good accuracy of the proposed method.

Precision: the precision is closeness or agreement of obtained analytical values to each other and is measured by

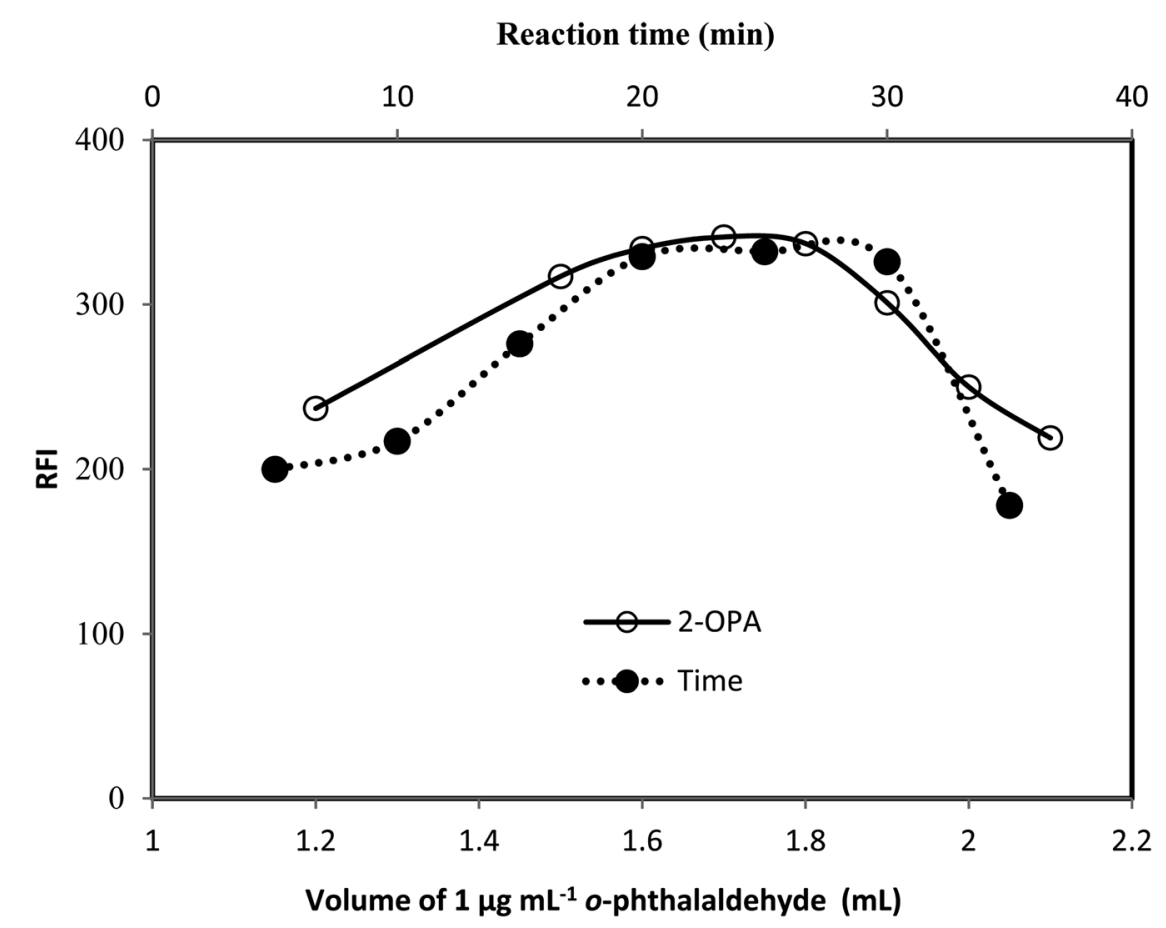

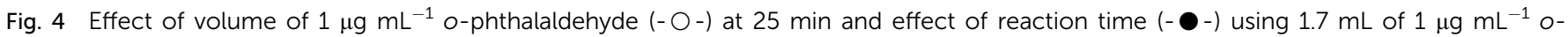

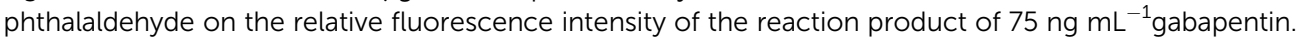




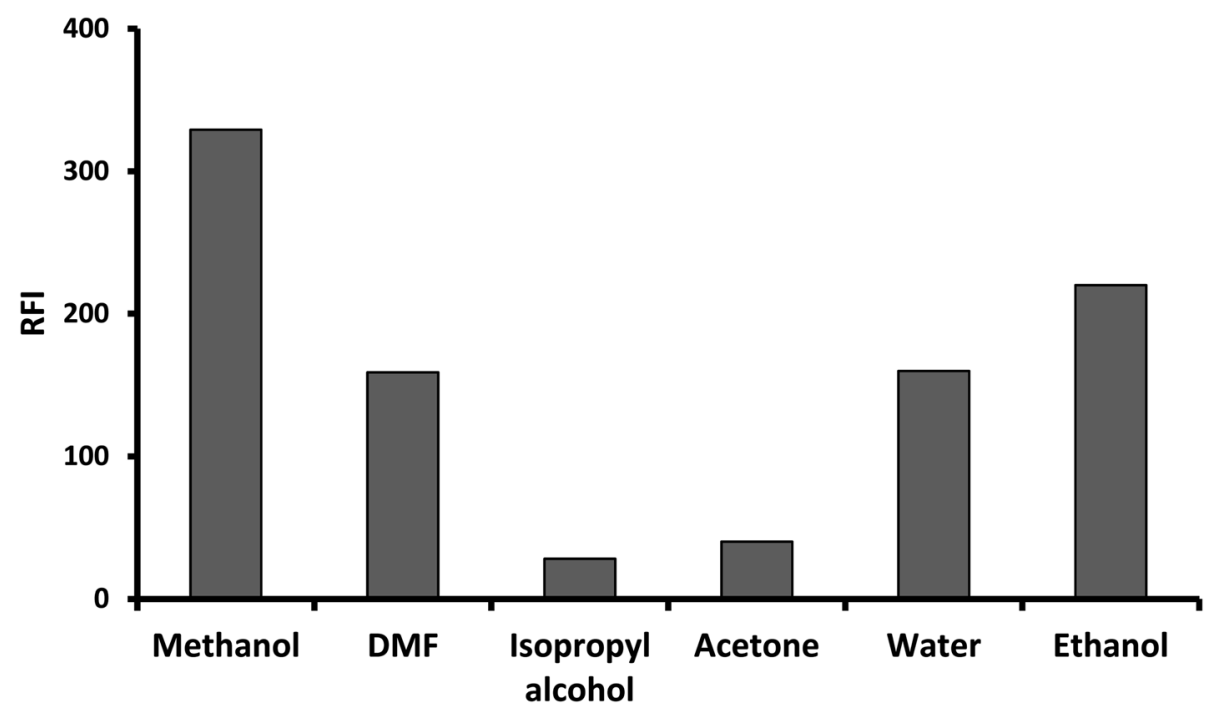

Fig. 5 Effect of different solvent on the relative fluorescence intensity of the reaction product of $75 \mathrm{ng} \mathrm{mL}^{-1}$ gabapentin.

Table 1 Analytical parameters for determination of the studied drug using the proposed method

\begin{tabular}{ll}
\hline Parameters & Value \\
\hline Linear range $\left(\mathrm{ng} \mathrm{mL}^{-1}\right)$ & $25-125$ \\
Intercept $(a)$ & 69.8 \\
Standard deviation of the slope $\left(S_{\mathrm{b}}\right)$ & 0.046 \\
Slope $(b)$ & 3.416 \\
Standard deviation of the intercept $\left(S_{\mathrm{a}}\right)$ & 3.849 \\
Correlation coefficient $(r)$ & 0.9997 \\
Limit of detection $\left(\mathrm{LOD}, \mathrm{ng} \mathrm{mL} \mathrm{mL}^{-1}\right)$ & 3.4 \\
Limit of quantitation $\left(\mathrm{LOQ}, \mathrm{ng} \mathrm{mL}^{-1}\right)$ & 11.2
\end{tabular}

calculating the relative standard deviation (RSD). The intra- and inter-day precisions were evaluated by applying the general analytical procedure for the analysis of standard drug solutions having three different concentrations within the specified linear range. The analysis was repeated three times within the same day for the intra-day precision and at three successive days for inter-day precision. The results were expressed in the form of $\%$ recovery and \% relative standard deviation (Table 3). The calculated relative standard deviations were found to be very

Table 2 Evaluation of accuracy for the proposed analytical method

\begin{tabular}{llcc}
\hline No. & Taken $\left(\mathrm{ng} \mathrm{mL}^{-1}\right)$ & Found $^{a}\left(\mathrm{ng} \mathrm{mL}^{-1}\right)$ & \% recovery \\
\hline 1 & 25 & 25.43 & 101.72 \\
2 & 75 & 74.41 & 99.21 \\
3 & 125 & 123.98 & 99.18 \\
Mean & & & 100.37 \\
$\mathrm{SD}^{b}$ & & & 1.458 \\
\% RSD $^{b}$ & & & 1.457
\end{tabular}

${ }^{a}$ The value is the average of three determinations. ${ }^{b} \mathrm{SD}$ is the standard deviation and \% RSD is the relative standard deviation percentage. small and below $2 \%$, indicating good repeatability and reliability of the proposed method.

Sensitivity: the sensitivity of the proposed method was evaluated by calculating the limit of detection (LOD) and limit of quantitation (LOQ). Based on the standard deviation of the intercept $(\sigma)$ and the slope of calibration curve $(S)$, the Limit of detection (LOD) and limit of quantitation (LOQ) were calculated using the following formula ( $\mathrm{LOD}=3 \sigma / S$ and $\mathrm{LOQ}=10 \sigma / S$ ). The obtained detection and quantitation limits were 3.4 and $11.2 \mathrm{ng}$ $\mathrm{mL}^{-1}$, respectively (Table 1 ). These values indicate the high sensitivity of the proposed method.

Robustness of the proposed method was evaluated by introducing minor changes in the experimental parameters such as volumes of $o$-phthalaldehyde, 2-mercaptoethanol, and sodium hydroxide and the reaction time (Table 5). These minor changes have no effect on the method performance as the obtained fluorescence intensities were nearly the same. As a result, the proposed method could be considered as robust.

\subsection{Application to pharmaceutical capsules}

The general analytical procedure was applied for the quantitative analysis of commercial capsules containing gabapentin. The obtained results were statistically compared with those of

Table 3 Evaluation for the precision of proposed method for determination of gabapentin

\begin{tabular}{|c|c|c|c|c|}
\hline \multirow{2}{*}{$\begin{array}{l}\text { Conc. level } \\
\left(\mathrm{ng} \mathrm{mL} \mathrm{m}^{-1}\right)\end{array}$} & \multicolumn{2}{|l|}{ Intra-day precision } & \multicolumn{2}{|l|}{ Inter-day precision } \\
\hline & $\%$ recovery $\pm \mathrm{SD}^{a}$ & $\% \mathrm{RSD}^{a}$ & $\%$ recovery $\pm \mathrm{SD}^{a}$ & $\% \mathrm{RSD}^{c}$ \\
\hline 25 & $98.59 \pm 1.17$ & 1.19 & $100.94 \pm 1.17$ & 1.15 \\
\hline 75 & $99.74 \pm 1.62$ & 1.63 & $98.96 \pm 1.62$ & 1.64 \\
\hline 125 & $100.28 \pm 1.17$ & 1.17 & $100.85 \pm 0.62$ & 0.61 \\
\hline
\end{tabular}

${ }^{a}$ SD - standard deviation, \% RSD - relative standard deviation percentage. 
Table 4 Analysis of gabapentin commercial capsule using the proposed spectrofluorimetric and reported methods

\begin{tabular}{|c|c|c|c|c|}
\hline Commercial capsules & \multicolumn{2}{|c|}{$\%$ recovery $\pm \operatorname{SD}(n=5)$} & $t$ value $^{a}$ & $F$ value $^{a}$ \\
\hline
\end{tabular}

Table 5 Robustness for the proposed analytical method

\begin{tabular}{lcr}
\hline Parameters & Change & \% recovery $\pm \mathrm{SD}$ \\
\hline$o$-Phthalaldehyde volume & $1.6 \mathrm{~mL}$ & $99.11 \pm 1.27$ \\
& $1.8 \mathrm{~mL}$ & $101.25 \pm 1.35$ \\
2-Mercaptoethanol volume & $0.4 \mathrm{~mL}$ & $99.32 \pm 1.16$ \\
& $0.8 \mathrm{~mL}$ & $101.45 \pm 1.23$ \\
Sodium hydroxide volume & $0.5 \mathrm{~mL}$ & $99.83 \pm 1.18$ \\
& $1.0 \mathrm{~mL}$ & $101.23 \pm 1.26$ \\
Reaction time & $20 \mathrm{~min}$ & $99.93 \pm 0.76$ \\
& $30 \mathrm{~min}$ & $101.33 \pm 0.87$
\end{tabular}

the reported method ${ }^{3}$ using $t$ - and $F$ - tests. At 95\% confidence level, no significant difference was observed between the calculated and theoretical values of both parameters indicating the acceptable level of precision and accuracy of the proposed method (Table 4). It is clear from the analysis of the dosage forms that the presence of excipients has no significant interference in the results of the analysis. This indicates that the proposed method could be used for determination of the investigated drug in their commercial capsules with a high degree of accuracy, precession and selectivity.

\section{Conclusion}

A highly sensitive procedure for the selective spectrofluorimetric determination of gabapentin through derivatization with $o$-phthalaldehyde was developed and validated. The proposed method was successfully applied for determination of investigated drug in their commercial pharmaceutical capsules with good accuracy and precision without any interference from common excipients. The involved methodology does not require elaborate treatment for the sample or tedious extraction steps. The high sensitivity together with the recognized advantage of the relatively low cost of spectrofluorometric instrumentation make the application of the proposed method is feasible in routine analysis. Therefore, the proposed method is ideally suited for determination of gabapentin in quality control laboratories.

\section{Conflicts of interest}

There are no conflicts to declare.

\section{References}

1 K. L. Goa and E. M. Sokrin, Gabapentin: A Review of Its Pharmacological Properties and Clinical Potential in Epilepsy, Drugs, 1993, 46, 409-427.

2 J. Al-Zehouri, S. Al-Madi and F. Belal, Determination of the antiepileptics vigabatrin and gabapentin in dosage forms and biological fluids using Hantzsch reaction, Arzneim. Forsch., 2001, 51, 97.

3 R. S. Gujral, S. M. Haque and P. Shanker, A Sensitive UV Spectrophotometric Method for the Determination of Gabapentin, E-J. Chem., 2009, 6(S1), S163-S170.

4 F. A. Siddiquia, et al., Spectrophotometric determination of gabapentin in pharmaceutical formulations using ninhydrin and $\pi$-acceptors, Eur. J. Med. Chem., 2010, 45(7), 2761-2767.

5 E. M. Hassan, et al., Spectrofluorimetric determination of vigabatrin and gabapentin in dosage forms and spiked plasma samples through derivatization with 4-chloro-7nitrobenzo-2-oxa-1,3-diazole, J. AOAC Int., 2001, 84, 1017.

6 F. Belal, et al., Spectrofluorimetric determination of vigabatrin and gabapentin in urine and dosage forms through derivatization with fluorescamine, J. Pharm. Biomed. Anal., 2002, 27, 253.

7 M. K. C. Prasad, G. V. Sagar and D. P. Sudhakar, Spectrofluorimetric method of analysis for gabapentin in spiked human plasma and formulations, Int. J. ChemTech Res., 2013, 5(6), 2732-2746.

8 S. T. Ulu and E. Kel, Sensitive Spectrofluorimetric Method of Analysis for Gabapentin in Pure and Pharmaceutical Preparations, Chin. J. Chem., 2011, 29, 562-566.

9 Q. Jiang and S. Li, Rapid high-performance liquid chromatographic determination of serum gabapentin, $J$. Chromatogr. B: Biomed. Sci. Appl., 1999, 727, 119.

10 P. H. Tang, et al., Automated microanalysis of gabapentin in human serum by high-performance liquid chromatography with fluorometric detection, J. Chromatogr. B: Biomed. Sci. Appl., 1999, 727, 125.

11 D. F. Chollet, et al., Fast isocratic high-performance liquid chromatographic assay method for the simultaneous determination of gabapentin and vigabatrin in human serum, J. Chromatogr. B: Biomed. Sci. Appl., 2000, 746, 311.

12 M. M. Kushnir, et al., Analysis of gabapentin in serum and plasma by solid-phase extraction and gas chromatographymass spectrometry for therapeutic drug monitoring, $J$. Anal. Toxicol., 1999, 23, 1. 
13 P. Rada, et al., In vivo monitoring of gabapentin in rats: A microdialysis study coupled to capillary electrophoresis and laser-induced fluorescence detection, Electrophoresis, 1998, 19, 2976.

14 A. Yari, F. Papi and S. Farhadi, Voltammetric Determination of Trace Antiepileptic Gabapentin with a Silver-Nanoparticle Modified Multiwalled Carbon Nanotube Paste Electrode, Electroanalysis, 2011, 23(12), 2949-2959.

15 A. El-Gindy, et al., Spectrophotometric,spectrofluorimetric and LC determination of lisinopril, J. Pharm. Biomed. Anal., 2001, 25, 913-922.

16 N. M. El-Enany, et al., Validated spectrofluorimetric method for the determination of lamotrigine in tablets and human plasma through derivatization with o-phthalaldehyde, $J$. Fluoresc., 2010, 20, 463-472.

17 M. I. Walash, et al., Spectrofluorimetric determination of oseltamivir phosphate through derivatization with ophthalaldehyde. Application to pharmaceutical preparations with a preliminary study on spiked plasma samples, Luminescence, 2012, 27, 511-518.

18 Validation of analytical procedures: methodology, Presented at international conference on harmonization of technical requirements for the registration of pharmaceuticals for human use (ICH), http:/www.fda.gov/downloads/drugs/ guidancecomplianceregulatoryinformation/guidances/ ucm073384.pdf, 1996. 